\section{Analysis of the Usage of Nanoscience and Technology in Chemistry}

The last few years have observed a wide proliferation of the terminology related to nanotechnology and nanoscience in chemistry. Today, all high-impact chemistry journals contain a large number of papers devoted to this growing area, as many conferences include specific sessions on nanotechnology.

The objective of this new project is to map and critically study the use of the prefix nano in various fields of chemistry. For this purpose, we will use the different search engines available to compare the usage of nano-containing terms. We will map the evolution and usage of nano-containing descriptive terms according to different criteria, and critically analyze their validity in scientific (chemical) language. This project is the first step towards recommendations on the use of chemistry terminology related to nanoscience and nanotechnology.

The methodology proposed is the following. First, we will use widely available and popular chemistry search engines, such as Sci Finder, and others provided by the CAS and RSC. The hits on "nano*" will be analyzed according to criteria, such as time, country, and source. Secondly, we will repeat the process by restricting the search to some of the most highly cited journals of each chemistry discipline, to learn if "nano-" terminology has impacted all the areas of chemistry and if so, to what extent and at what rate.

For more information and comments, please contact the Task Group Chair Javier Garcia Martinez <j.garcia@ua.es> or Sanjay Mathur <smathur@inm-gmbh.de〉.

www.iupac.org/projects/2007/2007-040-2-200.htm

\section{Extension of ThermoML-The IUPAC Standard for Thermodynamic Data Communications}

This project is an extension of project 2002-055-3024, XML-Based IUPAC Standard for Experimental and Critically Evaluated Thermodynamic Property Data Storage and Capture, which was successfully completed in 2006. From that project, a new XML-based IUPAC standard (ThermoML) was established for thermodynamic data communications (Pure Appl. Chem. 2006, 78, 541-612). Initially, ThermoML provided support of communications for experimental, critically evaluated, and predicted data for thermodynamic properties of pure and multi-component mixtures of molecular compounds with comprehensive representation of uncertainties (J. Chem. Eng. Data, 2003, 48, 2-13; 2003, 48, 1344-1359; and 2004, 49, 160174). Prior to the standard release, enhancements for aqueous electrolyte solutions and ionic liquids were included. The current project will broaden the scope of ThermoML to support storage and exchange of thermodynamic property data for speciation and complex equilibria in aqueous and non-aqueous solvents, and thermodynamic properties of biomaterials.

For more information and comments, please contact the Task Group Chair Michael Frenkel <frenkel@boulder.nist.gov>.

\section{www.iupac.org/projects/2007/2007-039-1-024.htm}

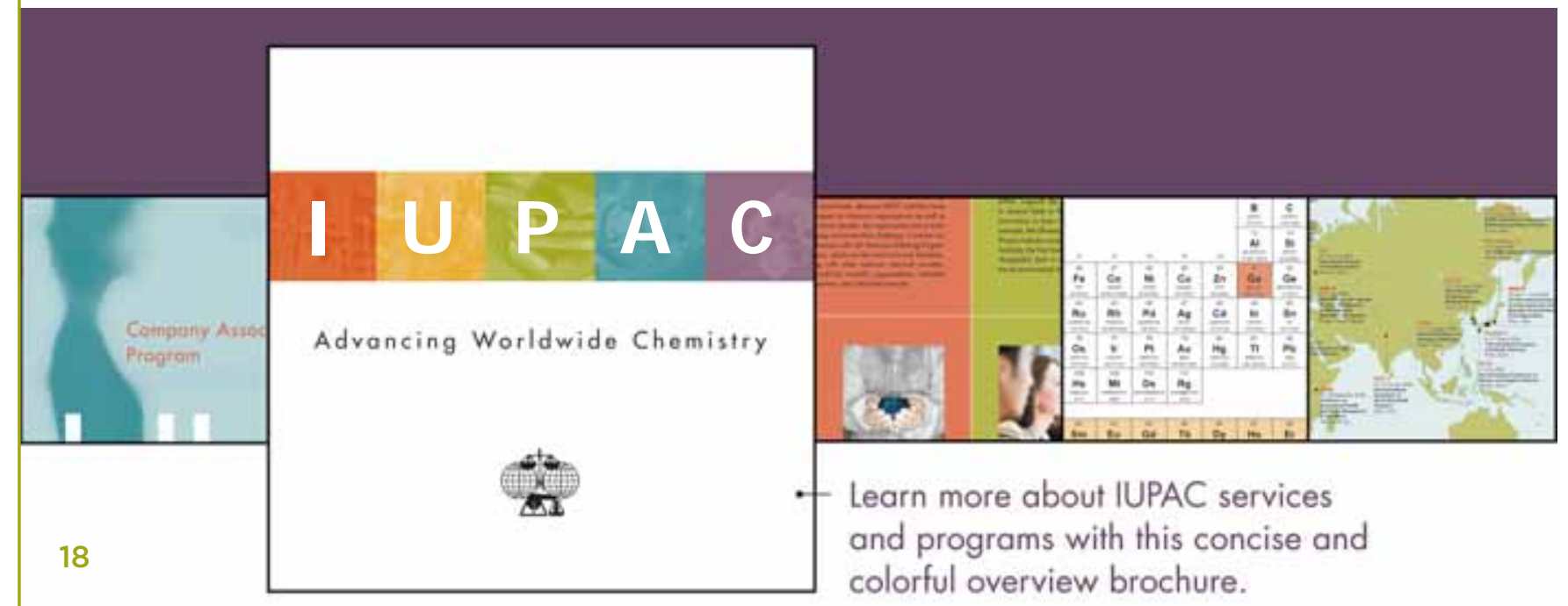

